\title{
Actitudes ambientales al final de la ESO. Un estudio diagnóstico con alumnos de Secundaria de la Región de Murcia
}

\author{
David Pérez-Franco ${ }^{1, a}$, Antonio José de Pro-Bueno ${ }^{1, b}$ Antonio Pérez-Manzano ${ }^{2, c}$ \\ ${ }^{1}$ Departamento de Didáctica de las Ciencias Experimentales, Facultad de Educación. Universidad de Murcia. \\ Murcia. España. \\ ${ }^{2}$ Departamento de Psicología Evolutiva y de la Educación. Facultad de Educación. Universidad de Murcia. \\ Murcia. España. \\ adavidperesf83@gmail.com, bnono@um.es, caperez@um.es
}

[Recibido: 5 Enero 2018. Revisado: 28 Abril 2018. Aceptado: 9 Julio 2018]

\begin{abstract}
Resumen: La educación ambiental debe fomentar la concienciación ambiental, generar actitudes favorables a la conservación del medio y al desarrollo sostenible, y contribuir a generar ciudadanos responsables y con un criterio sólido y propio a través de la alfabetización científica. En este artículo se han evaluado las actitudes ambientales de 690 alumnos murcianos de $4^{\circ}$ de educación secundaria obligatoria utilizando para ello el instrumento del proyecto ROSE. Además, se ha estudiado la relación con otras variables: el género, el nivel de estudios de los padres y la categoría profesional de estos últimos. Los resultados muestran una actitud moderadamente positiva con diferencias de género a favor de las chicas. Se observan también algunas tendencias -poco significativas- más favorables cuanto mayor sea el nivel de estudios de los padres y si la profesión está ligada al ámbito científico técnico. Finalmente, se propone utilizar las actitudes ambientales más positivas como vía para mejorar las actitudes hacia las ciencias y, en general, la educación científica.
\end{abstract}

Palabras clave: Actitudes ambientales, educación ambiental, ROSE, diferencias de género, educación secundaria.

Do environmental attitudes change in secondary education? A diagnostic study with Secondary students from Murcia

\begin{abstract}
Environmental education should raise environmental awareness, create favorable attitudes towards the preservation of the environment and sustainable development, and contribute to create responsible individuals with an independent judgement through scientific literacy. In this article the environmental attitudes of 690 students from Murcia in their fourth year of Secondary Education have been analyzed using ROSE project questionnaire. In addition, the connection between other variables such as gender and parents' both educational level and professional category has been considered. The results show a moderately positive attitude in general, although they revealed gender differences in favor of girls. We can also observe some favorable tendencies -less meaningful ones- when the parents have a higher educational level as well as when their profession is linked to the scientific technical area. Finally, it is suggested to use the most positive environmental attitudes as a tool to improve attitudes towards science and in general, towards scientific education.
\end{abstract}

Keywords: Environmental attitudes, environmental education, ROSE, gender differences, secondary school.

Para citar este artículo: Pérez-Franco D., de Pro-Bueno A., Pérez-Manzano A. (2018) Actitudes ambientales al final de la ESO. Un estudio diagnóstico con alumnos de Secundaria de la Región de Murcia. Revista Eureka sobre Enseñanzay Divulgación de las Ciencias 15 (3), 3501. doi: 10.25267/Rev_Eureka_ensen_divulg_cienc.2018.v15.i3.3501

\section{Introducción}

El estudio de las actitudes hacia el medio ambiente resulta interesante por la influencia de éstas sobre la conducta humana, ya que afecta al uso o abuso de los recursos naturales, a la conservación o deterioro de la calidad del medio o a un desarrollo sostenible o insostenible de la sociedad. El reto que plantea promover estilos de vida más proambientales es buscar la conexión de un modo eficiente que incorpore factores como la norma social, los valores, las actitudes, las creencias, el contexto y la conducta (De Castro 2001).

\author{
Revista Eureka sobre Enseñanza y Divulgación de las Ciencias \\ Universidad de Cádir. APAC-Eureka. ISSN: 1697-011X \\ bttp:/ / dx.doi.org/10.25267/Rev_Eureka_ensen_divulg_cienc.2018.v15.i3.3501 \\ bttp:/ / reuredc.uca.es
}


Las actitudes ambientales son de carácter complejo como demuestra la extensa bibliografía existente sobre el tema tanto dentro como fuera de nuestras fronteras. Franzen y Vogl (2013) realizaron un análisis comparativo en treinta y tres países (entre ellos España) comprendiendo veinte años de estudios sobre actitudes ambientales, concluyendo que dichas actitudes están disminuyendo progresivamente durante las últimas dos décadas en los países estudiados, sin encontrar una razón objetiva para tal declive.

En España se encuentran numerosos estudios de carácter empírico. Estos han intentado profundizar en la relación de las actitudes y conductas ambientales, así como en encontrar predictores de la conducta proambiental (Amérigo, Aragonés y García 2012, Amérigo y García 2014). De ellos se extraen algunas conclusiones interesantes: no existe un modelo único de predicción de la conducta ambiental (Berenguer y Corraliza 2000); no se han utilizado instrumentos homogéneos (Amérigo 2006); las actitudes y las conductas ambientales presentan correlaciones muy bajas; las actitudes tienen gran influencia sobre el comportamiento si otros factores no impiden que éste se materialice (Álvarez y Vega 2009); y por último, a las personas les cuesta actuar en favor del medio ambiente a pesar de reconocer la gravedad de los problemas (Moreno, Corraliza y Ruiz 2005).

$\mathrm{Si}$ el estudio de las actitudes es interesante por su influencia en la conducta humana, las que tienen los jóvenes tienen una mayor prioridad porque son más fáciles de modificar o adecuar. La educación obligatoria debería tener como objetivo el formento de individuos ambientalmente responsables que participen en una sociedad sostenible (ONU 1993, González 1996, Álvarez y Vega 2009). Por lo tanto, la importancia que adquiere la incorporación de la educación ambiental al currículum oficial radica en que su presencia puede determinar la trasmisión de las necesidades del mundo natural, la promoción de una alfabetización científica, el desarrollo de una educación relevante para la sociedad y la utilidad para resolver problemas en la vida diaria (Vázquez y Manassero 2005). Dicho currículum debe, además, inculcar un pensamiento crítico que forje un criterio propio y que a su vez provea al ciudadano de herramientas suficientes para actuar de forma adecuada ante las diferentes problemáticas ambientales (Novo 2005).

Existen importantes contribuciones sobre las actitudes ambientales de los jóvenes en el mundo educativo. En el ámbito internacional, por ejemplo, destacan las aportaciones del proyecto ROSE (Schreiner y Sjøberg 2004), que las estudia dentro del marco de las actitudes hacia la ciencia. En el ámbito nacional, se encuentran, por ejemplo, los estudios de Vázquez y Manasero (2004, 2005, 2008, 2009a) en los que muestran que las actitudes ambientales de los jóvenes son positivas, pero con algún aspecto pesimista: la predisposición a actuar en favor del medio ambiente (conducta ecológica) es baja en relación a la preocupación mostrada. Dichos resultados coinciden con otros, como el de Murga (2008), realizados también en este contexto.

Si se comparan con otros países, los jóvenes españoles se sitúan en la media en actitudes, son más pasivos y muestran más confianza en los expertos para solucionar los problemas (Vázquez y Manassero 2007a).

También se han realizado investigaciones longitudinales, en las que las actitudes ambientales se mantienen al mismo nivel desde primaria hasta secundaria en contraste con las actitudes hacia la ciencia escolar (Vázquez y Manassero 2008). Se detectan también diferencias en cuanto al género, más o menos pronunciadas, generalmente más favorables hacia el medio ambiente en las chicas que en los chicos (Vázquez y Manassero 2005, 2009b, Pérez 2012, Contreras 2012, Rivera, Calderón, Salazar y Sepúlveda 2016), llegándose a hablar de brecha de género en las actitudes ambientales (Bord y O'Connor 1997). Dos posturas se han planteado en la literatura para explicar dicha brecha: la que entiende que el rol social de la mujer, como cuidadora del grupo familiar, le acerca más a los recursos naturales y le dota de mayor sensibilidad 
(ecofeminismo socialista); y otra que considera que esa mayor sensibilidad procede de la naturaleza biológica diferencial de hombres y mujeres (ecofeminismo esencialista) (Sabaté 2000).

Otros trabajos apuntan a la carencia en los jóvenes de criterios y conocimientos científicos sólidos sobre esta temática (Murga 2008, Pérez 2012). Y es que el aprendizaje sobre las problemáticas del medio tiene una relación positiva con las actitudes ambientales (Benegas y Marcén 1995, Campbell, Waliczek y Zajicek 1999, Gifford y Nilsson 2014). De esta manera, consideramos que la educación ambiental debe afrontar la tarea de integrar complejos contenidos científicos sin caer en el reduccionismo (García 2004) y además complementarlos con una formación en valores, actitudes y normas (De Castro 2001, Vázquez y Manassero 2005). De ahí que deba formar parte de la propia educación o alfabetización científica (Sauvé 2010).

Sin embargo, las disciplinas científicas y la enseñanza de las ciencias se han centrado tradicionalmente más en otros aspectos curriculares (objetivos, contenidos, conocimientos, materiales, métodos y técnicas), dejando de lado su carácter instrumental para la educación en valores, conductas, toma de decisiones y en la formación de un espíritu crítico (Solbes y Vilches 2004, Vázquez y Manassero 2005). Este problema se acrecienta si tenemos en cuenta que, para una buena parte de los estudiantes, la educación obligatoria será su último contacto con la enseñanza reglada en ciencia y tecnología (Acevedo, Vázquez y Manassero 2003). Por tanto, necesita adquirir ese conocimiento (alfabetización científico-ambiental) en esta etapa educativa para poder acceder a una nueva cultura de consumo y de desarrollo (Álvarez y Vega 2009) y, además, para contribuir no sólo a la formación sino también a la identificación y la reflexión sobre los problemas existentes, a la participación en la toma de decisiones fundamentada y a la potenciación de su espíritu crítico (Gil y Vilches 2004).

En este escenario, los estudios sobre evaluación de actitudes ambientales en los últimos años en jóvenes son escasos a nivel nacional y sobre todo local. Debido a la velocidad a la que cambia la visión general de la sociedad y las constantes modificaciones de las leyes educativas, un nuevo estudio de las actitudes hacia el medio ambiente es algo siempre necesario. De esta manera se podrá aportar nueva información y nuevos datos para poder debatir y definir las líneas e iniciativas más adecuadas a seguir en la educación ambiental de nuestro sistema educativo. Por todo se plantea:

¿Qué actitudes ambientales tiene el alumnado al finalizar la ESO en la Comunidad Autónoma de la Región de Murcia? ¿Tiene relación con algunas variables de carácter social (género, estudios y profesiones de los padres)?

\section{Metodología}

\section{Participantes}

La población objeto del estudio es el alumnado del último curso de educación secundaria obligatoria (ESO) de la Región de Murcia. El cuestionario se aplicó en el primer semestre de 2015. Las edades del alumnado oscilan entre 15 y 18 años, siendo mayoritarios aquellos con 15-16 (81,3\%). El total de la muestra alcanza los 690 estudiantes, representando así un 4,69\% del total del alumnado de la Región de Murcia (muy superior al establecido por Krejcie y Morgan (1970) en sus trabajos sobre el tamaño de las muestras), procedentes de diferentes centros, los cuales se seleccionaron por su facilidad de acceso. En cuanto al género, las chicas conforman un 52,6\% de los participantes por un 47,4\% de los chicos. Otros descriptores de la muestra son el nivel de estudios y las profesiones de padres y madres. Estas últimas se han 
clasificado en base a la Clasificación Nacional de Ocupaciones (CNO-11). La muestra es heterogénea, ya que incluye alumnos que han optado por materias humanistas como científicas.

\section{Instrumento}

El instrumento de investigación utilizado es el cuestionario ROSE (Schreiner y Sjøberg 2004). Se ha seleccionado este instrumento por dos razones: en primer lugar se trata de un instrumento bastante homogéneo, y en segundo lugar, ofrece un abanico muy amplio de opciones tanto a nivel nacional como internacional con los que comparar los resultados, ya que el estudio original se realizó en más de 35 países que incluyeron territorios de Europa, Asia, África o Sudamérica, y que aún en los últimos años se ha seguido utilizando en países tan dispares como Francia (Le Hebel, Montpied y Fontanieu 2014), China (Yeung y Li 2015), o Emiratos Árabes (Yang, Badri, Al-Mazroui, Al-Rashedi y Nai 2017) . Este instrumento se encuentra formado por 19 cuestiones o ítems, cuya génesis y propiedades métricas pueden consultarse en Schreiner y Sjøberg (2004) y en Vázquez y Manassero (2007b).

Los ítems contienen frases o sentencias muy breves sobre las que los estudiantes anónimamente emiten una valoración cerrada sobre una escala Likert de cuatro opciones (de la 1 a la 4), que indica el grado de acuerdo/desacuerdo, grado de importancia o frecuencia. Se opta por la escala de cuatro opciones, así como eliminar la opción de «no lo sé» para motivar una respuesta más comprometida del alumno. Para ofrecer la posibilidad de una posición neutral, el cuestionario ofrece la posibilidad de no contestar a una cuestión si el alumno no desea responder, no entiende la pregunta o simplemente no tiene clara su opción.

La duración para completar el cuestionario se estima entre 5 y 10 minutos; en algunas ocasiones la encuesta ha sido administrada por el investigador y en otras por los profesores.

\section{Diseño de los ítems}

El cuestionario ROSE sigue las reglas de la investigación metodológica que se supone ha de seguir un buen cuestionario: preguntas cortas, palabras simples, no asumir muchos conocimientos sobre el tema, evitar dobles negaciones y preguntas inductivas, eludir preguntas intencionales o términos emocionales, prevenir respuestas socialmente correctas, no tocar temas delicados, etc. (Oppenheim 2000).

Algunos de estos problemas son particularmente importantes en el cuestionario ROSE, ya que el instrumento nace para ser usado en diferentes culturas, traducido a otras lenguas y respondido por estudiantes que puede que deban responder en un idioma diferente al de su lengua materna. El cuestionario pone especial interés en evitar preguntas repetitivas que puedan frustrar o irritar a los encuestados.

Bajo el encabezado «D» «Yo y los desafíos medioambientales» se dan las siguientes instrucciones: ¿hasta qué punto estás de acuerdo con las siguientes declaraciones acerca de los problemas con el medio ambiente? Marca la respuesta con una X en cada línea. Sí no entiendes la afirmación, déjala en blanco.

Todas las cuestiones se encuentran diseñadas para obtener datos e información sobre las actitudes de los alumnos de $4^{\circ}$ de la ESO respecto a los desafíos medioambientales. Una parte importante se centran en conocer en qué grado los alumnos se muestran optimistas y proactivos frente a los problemas medioambientales (D5, D6, D7, D9, D10, D12 y D14). Otra parte, en ponderar en qué grado se muestran indiferentes y pasivos frente a dichos problemas (D1, D3, D8, D11 y D13). Y las restantes en valorar los derechos de la naturaleza (D15, D16, y D18). 
Estos ítems están inspirados en la literatura en alineación, impotencia y falta de significado (Seeman 1959) y en las escalas de medición revisadas en Measures of Social Psychological Attitudes (Robinson, Shaver y Wrightsman 2013). Los ítems D15, D16 y D18 están adaptados a partir de un cuestionario internacional de valores y medio ambiente (Skjak y Boyum 1993).

Las respuestas a estos ítems aportan información sobre los valores culturales de los estudiantes, así como sobre sus motivaciones para actuar ecológicamente. Con las contestaciones de los estudiantes se pueden detectar estructuras básicas que puede haber detrás de sus actitudes hacia los problemas ambientales.

\section{Análisis de datos}

Para la tabulación y el análisis de datos se utiliza el paquete estadístico SPSS. Como variables grupales se consideran el género, el nivel de estudios de padres y madres, y su grupo profesional.

Se indica el índice de acuerdo para cada cuestión. Se obtiene calculando la diferencia de la suma de las dos puntuaciones más altas de la escala (bastante de acuerdo y totalmente de acuerdo) y las dos más bajas (nada de acuerdo y algo de acuerdo). Las puntuaciones directas se tratan como variable continua a través de la media ponderada y, debido a que las variables no presentan una distribución normal, se comparan mediante la prueba $U$ de Mann-Whitney y Kruskal-Wallis, tomando como criterio de significación estadística de diferencias el nivel $(\mathrm{p} \leq 0,05)$.

El análisis de regresión lineal múltiple se aplica mediante el procedimiento paso a paso del paquete SPSS, usando los criterios por defecto para la entrada o salida de los predictores de la ecuación de regresión.

\section{Resultados}

Los índices de acuerdo muestran una actitud moderadamente positiva por parte de los alumnos hacia el medio ambiente y también con moderadas esperanzas de mejora hacia el futuro (tabla 1).

Las afirmaciones con las que los jóvenes están más de acuerdo (índice de acuerdo más positivos) fueron:

D7. Todavía estamos a tiempo de encontrar soluciones a los problemas medioambientales $(82,9 \%)$.

D12. Creo que cada uno de nosotros puede contribuir significativamente a la protección del medio ambiente (81\%).

D10. La gente debería preocuparse más por la protección del medio ambiente (79,6\%).

D15. Los animales deberían tener el mismo derecho a la vida que las personas $(62,8 \%)$.

D2. Los problemas medioambientales hacen que el futuro del mundo parezca sombrío y sin esperanza $(58,1 \%)$.

Como puede verse, son afirmaciones que serían deseables desde la perspectiva de las actitudes medioambientales favorables.

Por el contrario, las cuestiones con las que están más en desacuerdo (índice de acuerdo más negativos) fueron:

D8. La gente se preocupa demasiado por los problemas ambientales $(-67,2 \%)$. 
D1. Las amenazas al medio ambiente no son mi problema $(-66,6 \%)$.

D13. Deberíamos dejar los problemas medioambientales a los expertos $(-65,4 \%)$.

D3. Los problemas medioambientales están exagerados (-55,3\%).

D11. La responsabilidad de resolver los problemas medioambientales del mundo es de los países ricos $(-26,8 \%)$.

Tabla 1. Estadísticos descriptivos de «Yo y los desafíos medioambientales»

\begin{tabular}{cccccc}
\hline Cuestiones & $\begin{array}{c}\text { Nada de } \\
\text { acuerdo (\%) }\end{array}$ & $\begin{array}{c}\text { Algo de } \\
\text { acuerdo (\%) }\end{array}$ & $\begin{array}{c}\text { Blumnos: } \mathbf{( N = 6 9 0 )} \\
\text { acuerdo (\%) }\end{array}$ & $\begin{array}{c}\text { Totalmente de } \\
\text { acuerdo (\%) }\end{array}$ & $\begin{array}{c}\text { Índice de } \\
\text { acuerdo (\%) }\end{array}$ \\
\hline D1 & 54,6 & 28,7 & 11,6 & 5,1 & $\mathbf{- 6 6 , 6}$ \\
D2 & 6,2 & 14,7 & 34,2 & 44,8 & $\mathbf{5 8 , 1}$ \\
D3 & 40,3 & 37,4 & 15,9 & 6,5 & $\mathbf{- 5 5 , 3}$ \\
D4 & 8,6 & 18,2 & 34,9 & 38,3 & $\mathbf{4 6 , 4}$ \\
D5 & 11 & 31,7 & 36,1 & 21,2 & $\mathbf{1 4 , 6}$ \\
D6 & 4,9 & 18 & 32,5 & 44,6 & $\mathbf{5 4 , 2}$ \\
D7 & 1,9 & 6,6 & 31,2 & 60,2 & $\mathbf{8 2 , 9}$ \\
D8 & 49,8 & 33,8 & 10,5 & 5,9 & $\mathbf{- 6 7 , 2}$ \\
D9 & 18,1 & 27,6 & 33 & 21,3 & $\mathbf{8 , 6}$ \\
D10 & 2,6 & 7,6 & 25 & 64,8 & $\mathbf{7 9 , 6}$ \\
D11 & 34,2 & 29,2 & 23,2 & 13,4 & $\mathbf{- 2 6 , 8}$ \\
D12 & 1,5 & 8 & 26,8 & 63,7 & $\mathbf{8 1}$ \\
D13 & 43,2 & 39,5 & 10,2 & 7,1 & $\mathbf{- 6 5 , 4}$ \\
D14 & 14,1 & 29,4 & 35,8 & 20,8 & $\mathbf{1 3 , 1}$ \\
D15 & 6,9 & 11,7 & 21,3 & 60,1 & $\mathbf{6 2 , 8}$ \\
D16 & 27 & 29,4 & 24,5 & 19 & $\mathbf{- 1 2 , 9}$ \\
D17 & 14,5 & 38 & 35,5 & 12 & $\mathbf{- 5}$ \\
D18 & 5,9 & 22,6 & 34,9 & 36,6 & $\mathbf{4 3}$ \\
D19 & 19,9 & 43,5 & 26,2 & $\mathbf{- 2 6}$ \\
\hline
\end{tabular}

Ahora bien, se ha de indicar que el desacuerdo con algunas cuestiones (y en concreto con estas últimas) muestra una actitud deseable respecto a los desafíos medioambientales. Las dos primeras obviamente son rechazables.

En las afirmaciones relativas a actitudes deseables (D5, D6, D7, D9, D10, D12 y D14) los alumnos manifiestan una actitud moderadamente proactiva o muy proactiva (D5 y D6) y una actitud moderadamente optimista o muy optimista (D7, D9, D10, D12 y D14). Destaca la diferencia entre la capacidad propia de ayudar al medio ambiente $(54,2 \%)$ y el sacrifico que están dispuestos a realizar (14,6\%); por tanto, un 39,8\% no estaría del todo dispuesto a hacer algo que le costara a pesar de reconocer su capacidad para mejorar el medio ambiente. Aun así, hay que puntualizar que sólo una minoría no estaría dispuesta a realizar ningún tipo de sacrificio (11\%). También se observan algunos índices moderados de acuerdo con algunas afirmaciones (D14 y D9).

Respecto a las actitudes indiferentes (D1, D3 y D8) o pasivas (D11 y D13), muestran su desacuerdo, lo cual denota una actitud contraria a la indiferencia y a la pasividad. El responsabilizar de la solución de los problemas ambientales a los países ricos, es la cuestión que muestra desacuerdo con menor intensidad $(-26,8 \%)$, mientras las restantes sobrepasan el $-50 \%$ de desacuerdo. 
Los resultados en cuanto a actitudes pesimistas (D2, D17 y D19) son muy diversos según la cuestión: una importante mayoría ve el futuro sombrío y sin esperanza $(58,1 \%)$; la mitad opina que casi toda la actividad humana es perjudicial para el medio ambiente con una pequeña inclinación al desacuerdo $(-5 \%)$; y una mayoría se encuentra en desacuerdo de que ciencia y tecnología afecten negativamente al medio ambiente $(-26,7 \%)$. Al no existir un claro posicionamiento en el grado de acuerdo, se considera que los participantes se muestran neutrales ante las actitudes pesimistas.

Los derechos de la naturaleza (D15, D16 y D18) muestran también resultados interesantes: que el mundo natural es sagrado y que los animales deberían tener el mismo derecho a la vida que las personas son ampliamente aceptadas (43\% y 62,8\% respectivamente); pero, por otro lado, el uso de animales en la experimentación para salvar vidas humanas obtiene sólo un desacuerdo del $-12,9 \%$. Esto implica que, aunque una gran mayoría se posiciona a favor de los derechos de la naturaleza, una porción importante los relega a un segundo plano cuando se contraponen a los del hombre (visión antropocéntrica).

Por último, la actitud hacia la ciencia y tecnología como solucionadora de los problemas medioambientales (D4) es también ampliamente aceptada (46,4\%); la confianza en dichas disciplinas puede considerarse deseable.

\section{Diferencias de género}

Existen diferencias en las respuestas según género (tabla 2). Se han recogido las medias de ambos colectivos, las totales y sus desviaciones, y la probabilidad de significación (p). Se señalan las afirmaciones con diferencias significativas; las que no lo son se indica "ns".

Tabla 2. Estadísticos y prueba de Mann-Whitney de «Yo y los desafíos medioambientales» por género.

\begin{tabular}{|c|c|c|c|c|c|c|c|}
\hline \multirow[t]{2}{*}{ Cuestiones } & \multicolumn{2}{|c|}{ Chicas } & \multicolumn{2}{|c|}{ Chicos } & \multicolumn{2}{|c|}{ Total } & \multirow{2}{*}{$\begin{array}{l}\text { Significación de } \\
\text { diferencias (U de } \\
\text { Mann-Whitney) }\end{array}$} \\
\hline & Media & $\sigma$ & Media & $\sigma$ & Media & $\sigma$ & \\
\hline D1 & 1,54 & 0,80 & 1,81 & 0,91 & 1,67 & 0,87 & 0,00 \\
\hline D2 & 3,17 & 0,91 & 3,19 & 0,88 & 3,18 & 0,90 & ns \\
\hline D3 & 1,82 & 0,87 & 1,95 & 0,91 & 1,88 & 0,89 & 0,04 \\
\hline D4 & 2,96 & 0,95 & 3,12 & 0,94 & 3,04 & 0,95 & 0,02 \\
\hline D5 & 2,78 & 0,91 & 2,57 & 0,93 & 2,68 & 0,93 & 0,01 \\
\hline D6 & 3,30 & 0,85 & 3,04 & 0,90 & 3,17 & 0,89 & 0,00 \\
\hline D7 & 3,53 & 0,68 & 3,46 & 0,73 & 3,50 & 0,71 & ns \\
\hline D8 & 1,62 & 0,81 & 1,83 & 0,91 & 1,72 & 0,87 & 0,00 \\
\hline D9 & 2,58 & 1,06 & 2,58 & 0,96 & 2,58 & 1,02 & ns \\
\hline D10 & 3,61 & 0,69 & 3,42 & 0,79 & 3,52 & 0,75 & 0,00 \\
\hline D11 & 2,02 & 1,01 & 2,32 & 1,04 & 2,16 & 1,04 & 0,00 \\
\hline D12 & 3,65 & 0,62 & 3,40 & 0,77 & 3,53 & 0,70 & 0,00 \\
\hline D13 & 1,68 & 0,83 & 1,95 & 0,90 & 1,81 & 0,88 & 0,00 \\
\hline D14 & 2,68 & 0,93 & 2,58 & 1,00 & 2,63 & 0,97 & ns \\
\hline D15 & 3,56 & 0,78 & 3,11 & 1,03 & 3,35 & 0,93 & 0,00 \\
\hline D16 & 2,25 & 1,06 & 2,48 & 1,07 & 2,36 & 1,07 & 0,01 \\
\hline D17 & 2,47 & 0,88 & 2,43 & 0,88 & 2,45 & 0,88 & ns \\
\hline D18 & 3,14 & 0,86 & 2,88 & 0,93 & 3,02 & 0,91 & 0,00 \\
\hline D19 & 2,27 & 0,88 & 2,27 & 0,90 & 2,27 & 0,89 & ns \\
\hline
\end{tabular}


Ellas se muestran generalmente más proactivas y optimistas en las cuestiones que representan estas actitudes (D5, D6, D7, D9, D10, D12 y D14), siendo las diferencias significativas en más de la mitad de las mismas.

En las actitudes sobre indiferencia y pasividad, ellos se muestran más indiferentes (D1, D3 y D8) y pasivos (D11 y D13) con diferencias significativas en todas. Responsabilizar de los problemas ambientales a los países ricos es la cuestión que presenta más discrepancias.

En las cuestiones con actitudes pesimistas (D2, D17 y D19), las diferencias entre sexos son mínimas y por tanto no significativas, mientras que en las relativas a los derechos de la naturaleza (D15, D16 y D18) ellas se posicionan de forma más favorable y significativa en todas las cuestiones.

Por último, los chicos confían más en la ciencia y tecnología para solucionar los problemas ambientales (D4), siendo también las diferencias significativas en cuanto al género.

\section{Diferencias por profesión de los padres}

Para contrastar la variable profesión de padres y madres se han considerado dos grupos profesionales: los que alguno de los progenitores trabaja en ámbito científico-técnico y los que sus progenitores trabajan en el ámbito de los trabajos manuales, o relacionados con el campo o la mar (MCM). Hemos destacado aquellas cuestiones que presentan diferencias significativas $(\mathrm{p} \leq 0,05)$ en alguno de los progenitores respecto a los ámbitos profesionales (tabla 3); en el resto de las cuestiones (14/19) no hay diferencias significativas en ninguno de los progenitores.

Tabla 3. Estadísticos descriptivos de «Yo y los desafíos medioambientales» por profesión de los padres.

\begin{tabular}{|c|c|c|c|c|c|c|}
\hline \multirow[b]{2}{*}{ Cuestiones } & \multirow[b]{2}{*}{ Progenitor } & \multicolumn{2}{|c|}{$\begin{array}{c}\text { Científico - Técnico } \\
\text { Madre }(\mathrm{N}=186) \\
\text { Padre }(\mathrm{N}=243)\end{array}$} & \multicolumn{2}{|c|}{$\begin{array}{c}\text { Manual/Campo/Mar } \\
\text { Madre }(\mathrm{N}=193) \\
\text { Padre }(\mathrm{N}=269)\end{array}$} & \multirow{2}{*}{$\begin{array}{l}\text { Significación de } \\
\text { diferencias (U de } \\
\text { Mann-Whitney) }\end{array}$} \\
\hline & & Media & $\sigma$ & Media & $\sigma$ & \\
\hline \multirow{2}{*}{ D3 } & Madre & 1,71 & 0,86 & 2,02 & 0,94 & 0,00 \\
\hline & Padre & 1,79 & 0,84 & 1,89 & 0,92 & ns \\
\hline \multirow{2}{*}{ D4 } & Madre & 3,17 & 0,89 & 2,98 & 0,99 & ns \\
\hline & Padre & 3,16 & 0,85 & 2,97 & 0,95 & 0,03 \\
\hline \multirow{2}{*}{ D5 } & Madre & 2,85 & 0,98 & 2,58 & 0,88 & 0,00 \\
\hline & Padre & 2,8 & 0,96 & 2,64 & 0,88 & 0,04 \\
\hline \multirow{2}{*}{ D8 } & Madre & 1,58 & 0,8 & 1,77 & 0,89 & 0,03 \\
\hline & Padre & 1,67 & 0,87 & 1,73 & 0,82 & ns \\
\hline \multirow{2}{*}{ D15 } & Madre & 3,34 & 0,99 & 3,38 & 0,92 & ns \\
\hline & Padre & 3,26 & 0,98 & 3,45 & 0,86 & 0,02 \\
\hline
\end{tabular}

Entre las cuestiones con diferencias significativas, hay dos en las que estar en desacuerdo implica una imagen positiva frente a los desafíos medioambientales (D3 y D8). En éstas se puede observar que los hijos de progenitores pertenecientes al ámbito científico-técnico muestran puntuaciones más favorables hacia el medio ambiente. En las demás, no se ciñen a un tipo de actitud concreta, sino que se reparten entre proactividad, indiferencia, derechos de la naturaleza y confianza en la ciencia y la tecnología.

Madres y padres tienen el mismo número de cuestiones con diferencias significativas (tres) coincidiendo solo en una (D5). En cuanto a la profesión materna, la mayor diferencia se 
observa en: «los problemas medioambientales están exagerados»; y en la paterna en la afirmación: «los animales deberían tener el mismo derecho a la vida que las personas».

Solamente dos cuestiones (D3 y D5) permanecerían significativas aplicando un riesgo menor $(\mathrm{p} \leq 0,01)$ y ambas sólo en cuanto a la profesión materna.

\section{Diferencias por estudios de los padres}

$\mathrm{Al}$ igual que en la profesión, la variable sobre los estudios de los padres contempla, además del nivel de formación, el género de los progenitores. De todas las cuestiones de que consta el bloque, sólo cinco muestran diferencias significativas, según el nivel de estudios de alguno de los progenitores (tabla 4).

Todas las cuestiones, salvo una, coinciden con las cuestiones significativas en relación a la profesión de los padres y, de la misma forma, el desacuerdo en dos de ellas muestra actitudes favorables al medio ambiente (D3 y D8). Igualmente, tampoco se ubican en un tipo de actitud concreta, repartiéndose en este caso entre proactividad, optimismo, indiferencia, derechos de la naturaleza y confianza en la ciencia y la tecnología.

Tabla 4. Estadísticos descriptivos de «Yo y los desafíos medioambientales» por estudios de los padres.

\begin{tabular}{|c|c|c|c|c|c|c|c|c|}
\hline Cuestiones & Progenitor & \multicolumn{2}{|c|}{$\begin{array}{c}\text { Sin estudios / } \\
\text { Primarios } \\
\text { Madre }(\mathrm{N}=126) \\
\text { Padre }(\mathrm{N}=148)\end{array}$} & \multicolumn{2}{|c|}{$\begin{array}{c}\text { Secundarios } \\
\text { Madre }(\mathrm{N}=317) \\
\text { Padre }(\mathrm{N}=287)\end{array}$} & \multicolumn{2}{|c|}{$\begin{array}{l}\text { Universitarios } \\
\text { Madre }(\mathrm{N}=223) \\
\text { Padre }(\mathrm{N}=212)\end{array}$} & $\begin{array}{c}\text { Significación } \\
\text { de diferencias } \\
\text { (Kuskal- } \\
\text { Wallis) }\end{array}$ \\
\hline \multirow{2}{*}{ D3 } & Madre & 2,02 & 0,97 & 1,93 & 0,92 & 1,7 & 0,78 & 0,04 \\
\hline & Padre & 1,88 & 0,95 & 1,96 & 0,89 & 1,74 & 0,83 & 0,02 \\
\hline \multirow{2}{*}{ D5 } & Madre & 2,52 & 0,93 & 2,66 & 0,93 & 2,8 & 0,9 & 0,02 \\
\hline & Padre & 2,59 & 0,93 & 2,72 & 0,93 & 2,72 & 0,9 & $\mathrm{~ns}$ \\
\hline \multirow{2}{*}{ D8 } & Madre & 1,86 & 0,97 & 1,76 & 0,89 & 1,56 & 0,74 & 0,01 \\
\hline & Padre & 1,75 & 0,9 & 1,76 & 0,88 & 1,6 & 0,81 & ns \\
\hline \multirow{2}{*}{ D10 } & Madre & 3,34 & 0,9 & 3,59 & 0,68 & 3,57 & 0,67 & 0,03 \\
\hline & Padre & 3,43 & 0,82 & 3,59 & 0,69 & 3,52 & 0,7 & ns \\
\hline \multirow{2}{*}{ D15 } & Madre & 3,33 & 0,91 & 3,45 & 0,87 & 3,22 & 1,02 & 0,03 \\
\hline & Padre & 3,42 & 0,86 & 3,38 & 0,89 & 3,25 & 1,04 & $\mathrm{~ns}$ \\
\hline
\end{tabular}

En las cuestiones en las que hay diferencias significativas, se dan en el nivel de estudios de las madres y tan sólo una en el nivel de estudios de los padres, coincidiendo ambos en una de ellas (D3).

Los resultados muestran que los hijos de progenitores con el menor nivel de estudios tienen las puntuaciones menos favorables al medio ambiente en tres de las cuestiones (D3, D5 y D8). Sin embargo, en la cuestión sobre el derecho a la vida de los animales (D15), las menores puntuaciones pertenecen a los hijos de progenitores con estudios universitarios. Y en la D10 los estudios secundarios los que indican una actitud más favorable en ambos progenitores.

\section{Relación entre actitudes y actividades de tiempo libre}

Se han seleccionado siete actividades para relacionar con las actitudes ambientales:, jugar a videojuegos, leer, realizar tareas escolares, salir con amigos, juegos y deportes al aire libre, ver televisión u otro tipo de medios audiovisuales, y navegar por internet, incluyendo redes 
sociales y whatsapp. Los resultados más destacados resultan de las dos primeras: jugar a videojuegos y leer (tabla 5).

Tabla 5. Valor del coeficiente beta en regresión lineal por pasos de «Yo y los desafíos medioambientales» por actividades de los jóvenes (jugar a videojuegos y leer).

\begin{tabular}{ccccccc}
\hline Cuestiones & \multicolumn{2}{c}{ Jugar a videojuegos } & \multicolumn{2}{c}{ Leer } & & \\
& $\begin{array}{c}\text { Coeficiente } \\
\text { beta }\end{array}$ & $\begin{array}{c}\text { Probabilidad } \\
\text { de } \\
\text { significación }\end{array}$ & $\begin{array}{c}\text { Coeficiente } \\
\text { beta }\end{array}$ & $\begin{array}{c}\text { Probabilidad } \\
\text { de } \\
\text { significación }\end{array}$ & $\mathbf{R}$ & $\mathbf{R}^{2}$ \\
\hline D1 & 0,137 & 0,000 & $-0,130$ & 0,001 & 0,189 & 0,036 \\
D2 & ns & ns & 0,096 & 0,013 & 0,139 & 0,019 \\
D3 & 0,124 & 0,001 & $-0,107$ & 0,006 & 0,165 & 0,027 \\
D4 & 0,101 & 0,01 & ns & ns & 0,125 & 0,016 \\
D5 & $-0,129$ & 0,01 & 0,192 & 0,000 & 0,231 & 0,053 \\
D6 & $-0,151$ & 0,000 & 0,134 & 0,001 & 0,221 & 0,049 \\
D8 & 0,093 & 0,015 & $-0,109$ & 0,04 & 0,228 & 0,052 \\
D10 & ns & ns & 0,088 & 0,022 & 0,208 & 0,043 \\
D11 & 0,126 & 0,001 & ns & ns & 0,126 & 0,016 \\
D12 & ns & ns & 0,099 & 0,01 & 0,159 & 0,025 \\
D13 & 0,088 & 0,023 & $-0,098$ & 0,012 & 0,163 & 0,026 \\
\hline
\end{tabular}

Según los datos obtenidos, jugar a videojuegos tendría una relación desfavorable con una actitud ambiental positiva (D1, D3, D4, D5, D6, D8, D11 y D13), mientras que leer presentaría una relación favorable con dicha actitud (D1, D2, D3, D5, D6, D8, D10, D12 y D13).

\section{Conclusiones}

Se observa una actitud moderadamente positiva hacia el medio ambiente y hacia la esperanza de mejora en el futuro, lo que concuerda con otros estudios similares del proyecto ROSE (Vázquez y Manassero 2005, 2009b, Schreiner 2006, Sjøberg y Schreiner 2008).

Si se contrastan nuestros resultados con los de estos estudios (figura 1), apreciando que son similares tanto a la media de países europeos como al estudio realizado en jóvenes baleares. En general, los estudiantes de Murcia revelan unas actitudes levemente más positivas que las Baleares y ambas más positivas que la media europea.

Específicamente, se detectan algunas diferencias en D2 (los problemas medioambientales hacen que el futuro del mundo parezca sombrío y sin esperanza), D4 (La ciencia y la tecnología pueden resolver problemas ambientales), D6 (yo puedo ayudar a mejorar el medio ambiente), D13 (deberíamos dejar los problemas medioambientales a los expertos) y D16 (estoy de acuerdo en usar animales en experimentos médicos si eso puede salvar vidas humanas). Así nuestra población se mostraría en estas cuestiones más pesimista, con más confianza en la Ciencia y la Tecnología, más proactiva, menos pasiva y más respetuosa con los derechos de los animales. 
D18. El mundo natural es sagrado y deberíamos.

D17. Casi toda la actividad humana es perjudicial..

D16. Estoy de acuerdo en usar animales en..

D15. Los animales deberían tener el mismo..

D14. Soy optimista sobre el futuro del medio..

D13. Deberíamos dejar los problemas..

D12. Creo que cada uno de nosotros puede..

D11. La responsabilidad de resolver los.

D10. La gente debería preocuparse más por la..

D9. Los problemas medioambientales pueden..

D8. La gente se preocupa demasiado por los..

D7. Todavía estamos a tiempo de encontrar.. D6. Yo puedo ayudar a mejorar el medio ambiente

D5. Estoy dispuesto a resolver los problemas.

D4. La ciencia y la tecnología pueden resolver.

D3. Los problemas medioambientales están..

D2. Los problemas medioambientales hacen que..

D1. Las amenazas al medio ambiente no son mi..

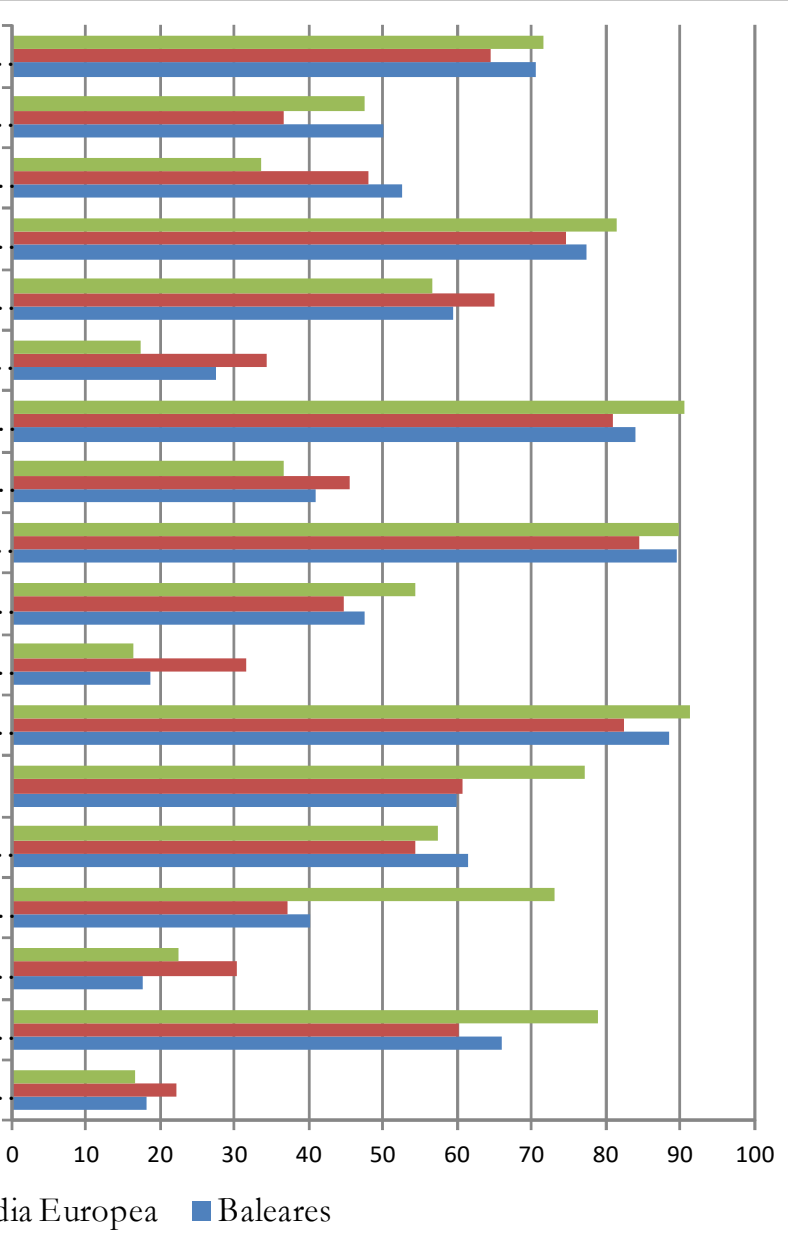

Figura 1. Comparación de respuestas favorables a las cuestiones «Yo y los desafíos medioambientales» entre Baleares, Murcia y la media Europea.

\section{Diferencias de género}

Confirmando otros hallazgos de la literatura (Trobat, Castells, Casero y Morey 2005, Vázquez y Manassero 2005, 2009b, Sjøberg y Schreiner 2008), nuestros resultados ponen de manifiesto unas actitudes más favorables en las chicas en la mayoría de las cuestiones, reflejando más responsabilidad, concienciación y respeto, aunque los de los chicos son también positivos en la mayoría.

Las actitudes en las que chicos y chicas presentan opiniones más discrepantes son las relativas a los derechos de la naturaleza, ya que en todas ellas hay diferencias significativas. Este aspecto debería tenerse en cuenta en la elección de los contenidos en la ciencia escolar, ya que la Biología y las Ciencias de la Salud gozan de mayor aceptación -y aún más entre las chicas- que la Química o la Física (Jenkins y Pell 2006).

Las cuestiones sobre el deterioro del medio ambiente por la actividad humana o por las actividades de la CyT no presentan casi diferencias, lo que nos indica que la percepción del problema es similar en ambos sexos, encontrándose la diferencia en el posicionamiento ante tales problemas y en la priorización en su escala de valores. De esta manera ellos adoptan actitudes de indiferencia y pasividad con mayor frecuencia que ellas.

En cuanto al pesimismo, no queda reflejado que las mujeres sean más pesimistas que los hombres como en otros estudios (Oscarsson 1996, Hutchinson 1997, Trobat et al. 2005). Ambos obtienen resultados muy similares en dichas cuestiones. Por el contrario, en nuestro 
estudio, resultan más proactivas mostrando una mayor predisposición a la acción y al sacrificio personal en favor del medio ambiente.

\section{Profesión y estudios de los padres}

La mayoría de las cuestiones no presenta diferencias ni en cuanto a profesión ni en cuanto a estudios, por lo que no parece un factor determinante a la hora de generar actitudes positivas (o negativas) hacia el medio ambiente. Esto no quiere decir que no sean influyentes, ya que se observa de forma general y en la mayoría de las cuestiones una tendencia de los alumnos a unas mayores puntuaciones en las actitudes optimistas, proactivas y pesimistas a mayor formación académica y también en profesiones cercanas a la ciencia y la tecnología.

Aunque sólo unas pocas resulten significativas, se detectan las diferencias más importantes cuando se trata de la madre. La mayor influencia de la madre sobre las actitudes de los alumnos coincide con un trabajo del Instituto Nacional de Evaluación Educativa sobre el informe PISA 2012 (INEE 2014). De esta manera, la mejor actitud de las mujeres hacia el medio ambiente (Vázquez y Manassero 2007a) influiría en mayor medida que la del padre en los hijos y de forma favorable al medio ambiente, siendo necesario investigar en un futuro esta diferencia parental de influencias.

\section{Relación entre actitudes y actividades de tiempo libre}

La relación positiva entre lectura y actitudes puede responder a un mayor nivel cultural de aquellos estudiantes con hábitos lectores que desemboque en la construcción de un criterio más sensibilizado con el medio ambiente.

Por otro lado, la relación negativa entre videojuegos y actitudes ambientales pone en evidencia una brecha entre actitudes hacia la tecnología y actitudes hacia el medio ambiente.

\section{Hipótesis de trabajo}

Las actitudes hacia el medio ambiente de los alumnos de secundaria en nuestro país, a pesar de ser moderadamente positivas, se encuentran estancadas. Hace una década que se realizaron los estudios del proyecto ROSE en España y dichas actitudes apenas han evolucionado. El cambio en las leyes educativas en el pasado (LOE, LOGSE, etc.) no ha tenido influencia y probablemente ocurra lo mismo con la LOMCE y otras futuras leyes mientras que se mantenga el sistema tradicional de enseñanza con predominio de los contenidos declarativos sobre las actitudes y emociones. Para una educación ambiental que no sólo genere actitudes, sino que estas se traduzcan en conductas ecológicas, se necesita de nuevas líneas de acción educativas que incluyan el papel de las normas, la estabilización de la actitud, la importancia de la experiencia previa y la accesibilidad de la actitud educada o creada (Vázquez y Manassero 2005).

Las diferencias de género encontradas sugieren que se deben hacer esfuerzos en las actitudes de los chicos, principalmente para que estos otorguen más valor al mundo natural. Las mejores actitudes en las chicas pueden ser de utilidad para motivar y mejorar la relación de las alumnas con el resto de disciplinas científicas en las cuales muestran un menor interés que los chicos (Vázquez y Manassero 2008).

El hecho de que las actitudes ambientales en ambos sexos no decaigan (como ocurre con las científicas) durante primaria y secundaria (Vázquez y Manassero 2008), debe servir de guía para tender puentes con otras actitudes procientíficas. Por tanto, dichas actitudes pueden convertirse en una herramienta de motivación que contribuya tanto a la alfabetización científica como a disminuir el declive vocacional científico en la juventud española. 
La ruptura entre jugar a videojuegos y las actitudes ambientales refleja que, lejos de aprovechar una herramienta como la tecnología para fomentar los valores ambientales, parece existir cierto antagonismo entre las actitudes tecnológicas y las ambientales. Teniendo en cuenta la crisis que atraviesan las actitudes hacia la ciencia y la tecnología (Vázquez y Manassero 2008), parece obvio que combinar elementos potencialmente motivadores de ambas disciplinas (como son los videojuegos y las actitudes ambientales) debería ejercer un efecto complementario en vez de antagónico sobre la generación de dichas actitudes.

Por otro lado, si la lectura fomenta de manera positiva la actitud, otra conclusión evidente sería el de fomentar la lectura para la mejora de las actitudes.

Por último, destacar la importancia de fomentar la citada alfabetización científica en la educación ambiental, no como objetivo final, sino como vehículo generador de actitudes ambientales y conductas ecológicas en el marco de una sociedad igualitaria, así como de ciudadanos responsables y con capacidad crítica formal.

\section{Referencias}

Acevedo J. A., Vázquez A., Manassero M. A. (2003) Papel de la educación CTS en una alfabetización científica y tecnológica para todas las personas. Revista Electrónica de Enseñanza de Las Ciencias 2(2), 80-111.

Álvarez P., Vega P. (2009) Actitudes ambientales y conductas sostenibles. Implicaciones para la educación ambiental. Revista de Psicodidáctica 14(2), 245-260.

Amérigo M. (2006) La investigación en España sobre actitudes proambientales y comportamiento ecológico. Medio Ambiente y Comportamiento Humano 7(2), 45-71.

Amérigo M., Aragonés J., García J. (2012) Exploring the dimensions of environmental concern: An integrative proposal. Psyecology 3(3), 299-311.

Amérigo M., García J. (2014) Perspectiva multidimensional de la preocupación por el medio ambiente. Relación entre dimensiones actitudinales y comportamientos. Psico 45(3), 406-414.

Benegas J., Marcén C. (1995) La Educación Ambiental como desencadenante del cambio de actitudes ambientales. Revista Complutense de Educación 6(2), 11-28.

Berenguer J. M., Corraliza J. A. (2000) Preocupación ambiental y comportamientos ecológicos. Psicothema 12(3), 325-329. http://www.psicothema.com/pdf/338.pdf

Bord R., O'Connor R. (1997) The Gender Gap in Environmental Attitudes: The Case of Perceived Vulnerability to Risk. Social Science Quarterly 78(4), 830-840.

Campbell J., Waliczek T. M., Zajicek J. M. (1999) Relationship Between Environmental Knowledge and Environmental Attitude of High School Students. The Journal of Environmental Education 30(3), 17-21.

Contreras S. (2012) Actitudes ambientales de los estudiantes de secundaria en Baja California: características personales y académicas asociadas. Universidad Autónoma de Baja California.

De Castro R. (2001) Naturaleza y funciones de las actitudes ambientales. Estudios de Psicología 22(1), 11-22. http://dx.doi.org/10.1174/021093901609569

Franzen A., Vogl D. (2013) Two decades of measuring environmental attitudes: A comparative analysis of 33 countries. Globlal Environmental Change 23(5), 1001-1008. 
Gifford R., Nilsson A. (2014) Personal and social factors that influencepro-environmental concern and behaviour: A review. International Journal of Psychology 49(3), 141-157.

Gil D., Vilches A. (2004) La contribución de la ciencia a la cultura ciudadana. Cultura y Educación 16(3), 259-272. http://dx.doi.org/10.1174/1135640042360924

González M. C. (1996) Principales tendencias y modelos de la Educación ambiental en el sistema escolar. Revista Iberoamericana de Educación (11), 13-74.

Hutchinson F. (1997) Our children's futures: are there lessons for environmental educators? Environmental Education Research 3(2), 189-201.

Instituto Nacional de Evaluación Educativa. (2014) Ocupaciones de los padres y PISA 2012: El caso de las CC.AA. Educainee (2).

Jenkins E. W., Pell R. G. (2006) "Me and the Environmental Challenges": A survey of English secondary school students' attitudes towards the environment. International Journal of Science Education 28(7), 765-780.

Krejcie R. V, Morgan D. W. (1970) Determining sample size for research activities. Educational and Psychological Measurement 30(3), 607-610.

Moreno M., Corraliza J. A., Ruiz J. P. (2005) Escala de actitudes ambientales hacia problemas específicos. Psicothema 17(3), 502-508.

Murga M. A. (2008) Percepciones, valores y actitudes ante el desarrollo sostenible . Detección de necesidades educativas en estudiantes universitarios. Revista Española de Pedagogía 66(240), 327-343.

Novo M. (2005) Educación ambiental y educación no formal: dos realidades que se realimentan. Revista de Educación (338), 145-165.

ONU. (1993) Conferencia sobre Medio Ambiente y Desarrollo. Documentos de la Cumbre de Río Vol 2. Madrid: Ministerio de Obras Públicas, Urbanismo y Medio Ambiente.

Oppenheim A. (2000) Questionnaire design interviewing and attitude measurement. Bloomsbury Publishing.

Oscarsson V. (1996) Pupils' views on the future in Sweden. Environmental Education Research 2(3), 261-277.

Pérez A. (2012) Actitudes bacia la Ciencia en Primaria y Secundaria. Universidad de Murcia.

Rivera L., Calderón N., Salazar B., Sepúlveda C. (2016) Efectos de la enseñanza interdisciplinaria en la educación ambiental sobre los conocimientos, valores y actitudes ambientales de estudiantes de segundo ciclo básico (Los Angeles, Región del Biobío, Chile) . Revista Complutense de Educación 27(3), 1139-1155.

Robinson J. P., Shaver P. R., Wrightsman L. S. (2013) Measures of Personality and Social Psychological Attitudes: Measures of Social Psychological Attitudes (Vol. 1). San Diego: Academic Press, INC.

Sabaté A. (2000) Género, Medio Ambiente y Acción política: un debate pendiente en la Geografía Actual. Anales de Geografía de La Universidad Complutense (20), 177-191.

Sauvé L. (2010) Educación científica y educación ambiental: un cruce fecundo. Enseñanza de las Ciencias 28(1), 5-18.

Schreiner C. (2006) Exploring a ROSE-Garden: Norwegian youth's orientations towards science - seen as signs of late modern identities. Realfagdidaktike. University of Oslo. 
Schreiner C., Sjøberg S. (2004) Sowing the seeds of ROSE: background, rationale, questionnaire development and data collection for ROSE (The Relevance of Science Education): a comparative study of students' views of science and science education. Acta Didactica. Oslo.

Seeman M. (1959) On the Meaning of Alienation. American Sociological Review 24(6), 783.

Sjøberg S., Schreiner C. (2008) Concerns for the Environment Data From Rose (The Relevance of Science Education).

Skjak K. K., Boyum B. (1993) Attitudes toward the environment 1993. Bergen: International Social Survey Programme.

Solbes J., Vilches A. (2004) Papel de las relaciones entre ciencia, tecnología, sociedad y ambiente en la formación ciudadana. Enseñanza de Las Ciencias 22(3), 337-348.

Trobat M., Castells M., Casero A., Morey M. (2005) Actitudes y percepción del medio ambiente en la juventud española. Madrid: Ministerio de Medio Ambiente.

Vázquez A., Manassero M. A. (2004) Imagen de la ciencia y la tecnología al final de la educación obligatoria. Cultura y Educación 16(4), 385-398.

Vázquez A., Manassero M. A. (2005) Actitudes de los jóvenes en relación con los desafíos medio-ambientales. Infancia y Aprendizaje 28(3), 309-327.

Vázquez A., Manassero M. A. (2007a) En defensa de las actitudes y emociones en la educación científica (II): evidencias empíricas derivadas de la investigación. Revista Eureka sobre Enseñanza y Divulgación de las Ciencias 4(3), 417-441.

Vázquez A., Manassero M. A. (2007b) La relevancia de la educación cientifica. Universitat Illes Balears.

Vázquez A., Manassero M. A. (2008) El declive de las actitudes hacia la ciencia de los estudiantes: un indicador inquietante para la educación científica. Revista Eureka sobre Enseñanza y Divulgación de las Ciencias 5(3), 274-292.

Vázquez A., Manassero M. A. (2009a) Factores actitudinales determinantes de la vocación científica y tecnológica en secundaria. Cultura y Educación 21(3), 319-330.

Vázquez A., Manassero M. A. (2009b) La relevancia de la educación científica: Actitudes y valores de los estudiantes relacionados con la ciencia y la tecnología. Enseñanza de las Ciencias 27(1), 33-48. 


\section{Anexo I}

\section{YO Y LOS DESAFÍOS MEDIOAMBIENTALES}

¿Hasta qué punto estás de acuerdo con las siguientes declaraciones acerca de los problemas con el medio ambiente (contaminación del aire y del agua, el uso excesivo de los recursos, el cambio climático, etc.)?

(Marca la respuesta con una X en cada línea. Sí no entiendes la afirmación, déjala en blanco)

\begin{tabular}{|l|cccc|}
\hline & $\begin{array}{l}\text { Nada de } \\
\text { acuerdo }\end{array}$ & & $\begin{array}{l}\text { Totalmente } \\
\text { de acuerdo }\end{array}$ \\
\hline $\begin{array}{l}\text { 1. Las amenazas al medio ambiente no } \\
\text { son mi problema }\end{array}$ & $\square$ & $\square$ & $\square$ & $\square$ \\
\hline $\begin{array}{l}\text { 2. Los problemas medioambientales } \\
\text { hacen que el futuro del mundo parezca } \\
\text { sombrío y sin esperanza }\end{array}$ & $\square$ & $\square$ & $\square$ & $\square$ \\
\hline $\begin{array}{l}\text { 3. Los problemas medioambientales } \\
\text { están exagerados }\end{array}$ & $\square$ & $\square$ & $\square$ & $\square$ \\
\hline $\begin{array}{l}\text { 4. La ciencia y la tecnología pueden } \\
\text { resolver problemas medioambientales }\end{array}$ & $\square$ & $\square$ & $\square$ & $\square$ \\
\hline $\begin{array}{l}\text { 5. Estoy dispuesto a resolver los } \\
\text { problemas medioambientales aunque } \\
\text { suponga hacer algún sacrificio }\end{array}$ & $\square$ & $\square$ & $\square$ & $\square$ \\
\hline $\begin{array}{l}\text { 6. Yo puedo ayudar a mejorar el medio } \\
\text { ambiente }\end{array}$ & $\square$ & $\square$ & $\square$ & $\square$ \\
\hline $\begin{array}{l}\text { 7. Todavía estamos a tiempo de } \\
\text { encontrar soluciones a los problemas } \\
\text { medioambientales }\end{array}$ & $\square$ & $\square$ & $\square$ & $\square$ \\
\hline $\begin{array}{l}\text { 8. La gente se preocupa demasiado por } \\
\text { los problemas medioambientales }\end{array}$ & $\square$ & $\square$ & $\square$ & $\square$ \\
\hline $\begin{array}{l}\text { 9. Los problemas medioambientales } \\
\text { pueden resolverse sin grandes cambios } \\
\text { en nuestro modo de vida }\end{array}$ & $\square$ & $\square$ & $\square$ & $\square$ \\
\hline $\begin{array}{l}\text { 10. La gente debería preocuparse más } \\
\text { por la protección del medio ambiente }\end{array}$ & $\square$ & $\square$ & $\square$ & $\square$ \\
\hline $\begin{array}{l}\text { 11. La responsabilidad de resolver los } \\
\text { problemas medioambientales del mundo } \\
\text { es de los países ricos }\end{array}$ & $\square$ & $\square$ & $\square$ & $\square$ \\
\hline $\begin{array}{l}\text { 12. Creo que cada uno de nosotros } \\
\text { puede contribuir significativamente a la } \\
\text { protección del medio ambiente }\end{array}$ & $\square$ & $\square$ & $\square$ & $\square$ \\
\hline $\begin{array}{l}\text { 13. Deberíamos dejar los problemas } \\
\text { medioambientales a los expertos }\end{array}$ & $\square$ & $\square$ & $\square$ & $\square$ \\
\hline $\begin{array}{l}\text { 14. Soy optimista sobre el futuro del } \\
\text { medio ambiente }\end{array}$ & $\square$ & $\square$ & $\square$ & $\square$ \\
\hline
\end{tabular}




\section{CUESTIONES GENERALES SOBRE NUEVAS TECNOLOGÍAS}

(Marca la respuesta con una X en cada línea. Sí no entiendes la afirmación, déjala en blanco)

1. ¿Con qué frecuencia realizas las siguientes actividades?

\begin{tabular}{|l|c|c|c|c|c|}
\hline & $\begin{array}{c}\text { Varias } \\
\text { veces } \\
\text { al día }\end{array}$ & $\begin{array}{c}\text { Todos } \\
\text { los } \\
\text { días }\end{array}$ & $\begin{array}{c}\text { Algunas } \\
\text { veces a } \\
\text { la } \\
\text { semana }\end{array}$ & $\begin{array}{c}\text { Algunas } \\
\text { veces al } \\
\text { mes }\end{array}$ & Nunca \\
\hline $\begin{array}{l}\text { 1. Realizar tareas escolares (hacer } \\
\text { los deberes) }\end{array}$ & $\square$ & $\square$ & $\square$ & $\square$ & $\square$ \\
\hline $\begin{array}{l}\text { 2. Navegar por internet (redes } \\
\text { sociales, whatsapp, etc.) }\end{array}$ & $\square$ & $\square$ & $\square$ & $\square$ & $\square$ \\
\hline 3. Jugar a videojuegos & $\square$ & $\square$ & $\square$ & $\square$ & $\square$ \\
\hline 4. Leer & $\square$ & $\square$ & $\square$ & $\square$ & $\square$ \\
\hline 5. Ver audiovisuales o televisión & $\square$ & $\square$ & $\square$ & $\square$ & $\square$ \\
\hline 6. Salir con amigos & $\square$ & $\square$ & $\square$ & $\square$ & $\square$ \\
\hline 7. Juegos o deportes al aire libre & $\square$ & $\square$ & $\square$ & $\square$ & $\square$ \\
\hline
\end{tabular}

